This item was submitted to Loughborough's Institutional Repository (https://dspace.lboro.ac.uk/) by the author and is made available under the following Creative Commons Licence conditions.

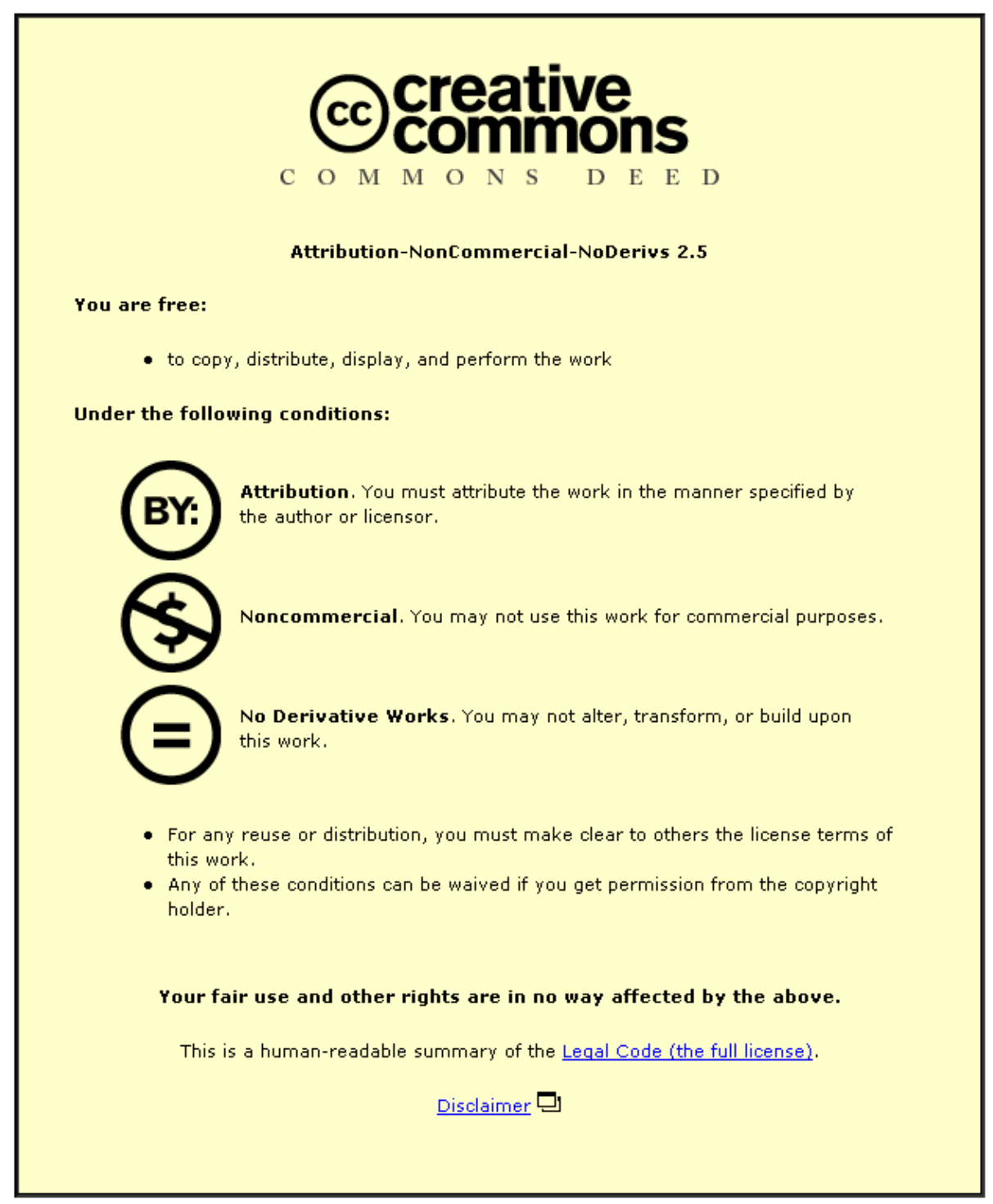

For the full text of this licence, please go to: http://creativecommons.org/licenses/by-nc-nd/2.5/ 


\title{
Importance Measures for Non-Coherent-System Analysis
}

\author{
Sally Beeson and John D. Andrews
}

\begin{abstract}
Component importance analysis is a key part of the system reliability quantification process. It enables the weakest areas of a system to be identified and indicates modifications, which will improve the system reliability. Although a wide range of importance measures have been developed, the majority of these measures are strictly for coherent system analysis. Non-coherent systems can occur and accurate importance analysis is essential. This paper extends four commonly used measures of importance, using the noncoherent extension of Birnbaum's measure of component reliability importance. Since both component failure and repair can contribute to system failure in a noncoherent system, both of these influences need to be considered. This paper highlights that it is crucial to choose appropriate measures to analyze component importance. First the aims of the analysis must be outlined and then the roles that component failures and repairs can play in system state deterioration can be considered. For example, the failure/repair of components in safety systems can play only a passive role in system failure, since it is usually inactive, hence measures that consider initiator importance are not appropriate to analyze the importance of these components. Measures of importance must be chosen carefully to ensure analysis is meaningful and useful conclusions can be drawn.
\end{abstract}

Index Terms-Fault tree, importance measure, noncoherent, structure function.

\section{ACRONYMS $^{1}$}

B\&P Barlow and Proschan

C controller

CS cut set

FC failure critical

FT fault tree

FV Fussell-Vesely

I1 ignition source

IV isolation valve

LF leaking flange

LP leaking pipe

N/A not applicable

PRV pressure-relief valve

$\mathrm{RC}$ repair critical

$s^{-} \quad$ statistical(ly)

SF $\quad$ system failure

SWS system is in a working state

Manuscript received June 8, 2001; revised June 1, 2002. Responsible Editor: J. H. Lambert.

The authors are with the Department of Systems Engineering, Loughborough University, Loughborough LE11 3TU, U.K. (e-mail: J.D.Andrews@lboro.ac.uk).

Digital Object Identifier 10.1109/TR.2003.816397

${ }^{1}$ The singular and plural of an acronym are always spelled the same.

$C_{k}$
$G_{i}(\underline{q})$
$G_{i}^{F}(\underline{q})$
$G_{i}^{R}(\underline{q})$
$G_{i, j}(\underline{q})$
$G_{i, \bar{j}}(\underline{q})$
$G_{\bar{i}, j}(\underline{q})$
$G_{\bar{i}, \bar{j}}(\underline{q})$
$G_{M_{i, j}}(\underline{q})$

\section{NOTATION}

prime-implicant set $k$ for noncoherent systems; minimal CS $k$ for coherent systems

Birnbaum measure of component-reliability importance

component-failure criticality: $\operatorname{Pr}\{$ SWS such that failure of component $i$ causes SF $\}$

component-repair criticality: $\operatorname{Pr}\{$ SWS such that repair of component $i$ causes SF $\}$

$\operatorname{Pr}\{$ SWS such that components $i$ and $j$ are FC $\}$ $\operatorname{Pr}\{$ SWS such that component $i$ is FC and component $j$ is RC

$\operatorname{Pr}\{$ SWS such that component $i$ is RC and component $j$ is FC

$\operatorname{Pr}\{$ SWS such that components $i$ and $j$ are RC $\}$

$\operatorname{Pr}\{$ SWS such that components $i$ and $j$ are FC, and the failure of either $i$ or $j$ is sufficient to cause SF $\}$

$G_{M_{i, j}}(\underline{q}) \quad \operatorname{Pr}\{$ SWS state such that component $i$ is FC and component $j$ is $\mathrm{RC}$, and the failure of $i$ or the repair of $j$ alone is sufficient to cause SF $\}$

$G_{M_{\bar{i}, j}}(\underline{q}) \quad \operatorname{Pr}\{$ SWS such that component $i$ is RC and component $j$ is $\mathrm{FC}$, and the repair of $i$ or the failure of $j$ alone is sufficient to cause SF $\}$

$G_{M_{i, j}}(\underline{q}) \quad \operatorname{Pr}\{$ SWS such that components $i$ and $j$ are RC, and the repair of either $i$ or $j$ alone is sufficient to cause SF \}

$\phi(\underline{x}) \quad$ structure function: system state in terms of the system components-states

$I_{C_{i}}^{F} \quad$ component-failure criticality measure

$I_{C_{i}}^{R^{i}}$

$I_{\mathrm{FV}}$

$I_{\mathrm{FV}_{i}}^{F}$

$I_{\mathrm{FV}_{i}}^{R}$

$I_{\mathrm{IN}_{i}}$

$I_{\mathrm{IN}_{i}}^{F}$

$I_{\mathrm{IN}_{i}}^{R}$

$I_{E_{i}}$

$I_{E_{i}}^{F}$

$I_{E_{i}}^{R}$

$\lambda$

$n_{p}$

$n_{c}$

$n$

$n_{\text {FI }}$

$n_{\mathrm{RI}}$ component-repair criticality measure

FV of component importance

FV measure of component-failure importance

FV measure of component-repair importance

$\mathrm{B} \& \mathrm{P}$ measure of initiator importance

Component-Initiator failure importance

Component-Initiator repair importance

Component-Enabler importance

component-failure enabler importance

component-repair enabler importance

failure rate

total number of prime-implicant sets or minimal CS

total number of components in prime-implicant set

total number of components in a system

total number of component-failure initiators

total number of component-repair initiators 


$\begin{array}{ll}p_{i} & p_{i}(t): \text { working probability of component } i \\ q_{i} & q_{i}(t): \text { failure probability of component } i \\ q_{\mathrm{Av}} & \text { average unavailability } \\ Q_{\mathrm{Sys}}(t) & \text { system unavailability function: } \operatorname{Pr}\{\text { system is in } \\ & \text { a failed state at time } t\} \\ Q_{M}(t) & \text { modified unavailability function } \\ Q\left(1_{i}, q(t)\right) & \operatorname{Pr}\{\text { system fails with component } i \text { failed }\} \\ Q\left(0_{i}, \underline{q}(t)\right) & \operatorname{Pr}\{\text { system fails with component } i \text { working }\} \\ \tau & \text { mean time to repair } \\ \theta & \text { test or inspection interval } \\ W_{\text {Sys }}(0, t) & \text { mean number of SF in }[0, t) \\ w_{i} & w_{i}(t): \text { unconditional failure intensity of compo- } \\ & \text { nent } i \\ v_{i} & v_{i}(t): \text { unconditional repair importance of compo- } \\ & \text { nent } i \text { failure }\end{array}$

\section{INTRODUCTION}

$\mathbf{R}$ ISK and reliability assessment techniques have developed over many years to meet the needs of industries such as the nuclear and oil industries; failure of safety systems in a nuclear power plant or on an offshore platform can have catastrophic consequences. Many regulatory bodies have now incorporated quantified risk assessment into safety requirements, to ensure that the risk of SF is as low as practicable.

Many techniques for risk and reliability assessment have been developed. One of the most popular is FT Analysis [1], [2], a deductive technique involving both qualitative and quantitative analysis. Qualitative analysis identifies the minimal CS, (or prime implicant sets in the case of noncoherent systems). Quantitative analysis involves quantification of the system unavailability and unreliability parameters and the analysis of component and/or minimal cut-set importance.

A FT is constructed by linking component states (usually failure modes) by gates (logical operators). The fundamental gates used during construction are AND gate, OR gate, and NOT gate. The construction process determines whether the FT structure is coherent, containing only AND gates and OR gates, or potentially noncoherent if it contains the NOT gate. A structure is noncoherent when its $\phi(\underline{x})$ does not comply with the following definition of coherency [3], [4]:

1) Every component $i, i=1, \ldots, n$ is relevant to the system state:

$$
\phi\left(1_{i}, \underline{x}\right) \neq \phi\left(0_{i}, \underline{x}\right) \text { for some } \underline{x} .
$$

2) The structure function is monotonically increasing (nondecreasing)

$$
\begin{aligned}
\phi\left(1_{i}, \underline{x}\right) & \geq \phi\left(0_{i}, \underline{x}\right) \quad \forall i ; \\
\phi\left(1_{i}, \underline{x}\right) & \equiv \phi\left(x_{1}, \ldots, x_{i-1}, 1, x_{i-1}, \ldots, x_{n}\right), \\
\phi\left(0_{i}, \underline{x}\right) & \equiv \phi\left(x_{1}, \ldots, x_{i-1}, 0, x_{i-1}, \ldots, x_{n}\right) .
\end{aligned}
$$

Andrews [5] demonstrated that for systems, such as multitasking systems, the use of NOT logic was essential for a meaningful analysis. The consideration of NOT logic during FT construction often results in a noncoherent FT. Numerous algorithms for identifying the prime implicant sets of the FT, and more recently techniques enabling system unavailability and un- reliability to be calculated, have been developed for this circumstance [6], [7]. However, importance analysis of noncoherent FT has received little attention. [8] attempts to extend some measures of importance; however, this extension of Birnbaum's measure was shown to be conceptually incorrect, rendering the extension of the other measures, based on this measure, incorrect. [9] extends Birnbaum's measure of importance to enable analysis of noncoherent systems.

This paper provides noncoherent extensions to 4 commonly used measures of importance; 3 of these measures are based on Birnbaum's measure of importance: component criticality, B\&P measure of Initiator importance, and Lambert's measure of Enabler importance. The fourth measure to be extended is the FV measure of component importance.

When the various extensions are introduced, a simple system with 5 prime implicants is used to demonstrate how these measures are calculated. These 5 prime implicants are: $\{a b d, a \bar{b} c, \bar{c} d e, a d e, a c d\}$

The Boolean expression obtained from these prime implicants is:

$$
T=a \cdot b \cdot d+a \cdot \bar{b} \cdot c+\bar{c} \cdot d \cdot e+a \cdot d \cdot e+a \cdot c \cdot d .
$$

The system unavailability can be obtained using the method outlined in [6]:

$$
\begin{aligned}
Q_{S y s}(t)= & q_{a} \cdot q_{b} \cdot q_{d}+q_{a} \cdot p_{b} \cdot q_{c}+p_{c} \cdot q_{d} \cdot q_{e} \\
& +q_{a} \cdot q_{d} \cdot q_{e}+q_{a} \cdot q_{c} \cdot q_{d}-q_{a} \cdot q_{b} \cdot q_{c} \cdot q_{d} \\
& -q_{a} \cdot q_{b} \cdot q_{d} \cdot q_{e} \\
& -q_{a} \cdot p_{b} \cdot q_{c} \cdot q_{d}-q_{a} \cdot p_{c} \cdot q_{d} \cdot q_{e} \\
& +q_{a} \cdot q_{b} \cdot q_{c} \cdot q_{d} \cdot q_{e} .
\end{aligned}
$$

\section{MEASURES OF IMPORTANCE}

Importance analysis is a part of the system quantification process which enables the analyst to rank the contribution that each component provides to SF, and thus identify the weakest areas of the system. Efforts to improve reliability can then be concentrated on those areas whose contribution indicates that by upgrading them, the maximum improvement in system reliability can be achieved. Importance measures assign a numerical value between 0 and 1 to each system component or minimal CS; the 1 signifies the highest level of importance. This signifies system susceptibility to component or minimal cut-set failure. Numerous measures of importance have been developed to enable analysts to assess the roles a component failure can play in the deterioration of the system state. For example, the B\&P Initiator importance measure considers the role that component failures play in causing SF, whereas the Lambert Enabler importance measure is concerned with the indirect role that a component failure plays in allowing other components to cause SF.

Measures of importance can be categorized as either deterministic or probabilistic. Deterministic measures assess the importance of a component or minimal cut-set without considering component reliability. Although these measures can be useful early in the design phase when information concerning component-failure probability is limited, generally probabilistic measures of importance are preferred because they provide morevaluable information. 
Probabilistic measures of importance can be further categorized as either 'measures concerned with system unreliability (contributing to failure frequency)' or 'measures concerned with system unavailability (contributing to failure probability)'. There is a clear and important distinction between the information the various measures of importance provide; consequently, measures must be chosen to suit the objectives of the study being performed.

Reliability $\equiv \operatorname{Pr}\{$ an item, system, or component operates without failure for a stated period of time, under specified conditions \}.

Availability $\equiv \operatorname{Pr}\{$ an item, system, or component can perform its required function at a particular time $\}$.

System availability is only of interest when SF can be tolerated; for hazardous industries, SF can be catastrophic; in such cases system unreliability must be assessed as opposed to system unavailability. For reliable systems, the unreliability can be approximated by the $s$-expected number of SF.

\section{BIRNBAUM's MEASURES AND ITS EXTENSION FOR NON-COHERENT ANALYSIS}

Birnbaum introduced a probabilistic measure of component reliability importance [10]: $\operatorname{Pr}\{$ component $i$ is critical to SF \}$=\operatorname{Pr}\{$ the system is residing in a critical state for a component, such that its failure causes SF $\}$ :

$$
G_{i}(\underline{q})=Q_{S y s}\left(1_{i}, \underline{q}(t)\right)-Q_{S y s}\left(0_{i}, \underline{q}(t)\right)=\frac{\partial Q_{S y s}(t)}{\partial q_{i}(t)} .
$$

The system unavailability can be calculated using the method outlined in [6]. In addition to providing an important measure in its own right, this measure also forms the basis for other measures of importance, including the measure of component criticality, B\&P measure of component Initiator importance, and Lambert's measure of component Enabler importance.

When analyzing noncoherent FT, both the 'component failed states' and 'component working states' can contribute to SF. Hence failed and working states occur in the prime implicant sets and consequently in the system unavailability function. Thus, to calculate $\operatorname{Pr}\{$ a component is critical to $\mathrm{SF}\}$, it is necessary to consider both component-failure and component-repair. Birnbaum's failure (repair) criticality for component $i$ is calculated by considering only the role that event $i$, and event $\bar{i}$, have in SF;

- event $i \equiv$ the failed state of component $i$,

- event $\bar{i} \equiv$ the working state of component $i$.

In mathematical equations,

$p_{i} \equiv \operatorname{Pr}\{$ a component works $\}$. and can be either reliability or availability,

$q_{i} \equiv \operatorname{Pr}\{$ a component does not work $\}$, and can be either unreliability or unavailability.

[9] develops an extension of Birnbaum's measure for the analysis of noncoherent systems. From this, expressions for the failure criticality, repair criticality, and total criticality, can be obtained: (4)-(6). These equations hold provided that Henley and Inagaki's method was used to calculate $Q_{S y s}(t)$. A component is $\mathrm{FC}$ at time $t$ if and only if the system is in a working state such that the failure of component $i$ causes the system to fail.

$$
G_{i}^{F}(\underline{q})=\frac{\partial Q_{S y s}(t)}{\partial q_{i}} .
$$

Similarly a component is RC at time $t$ if and only if the system is in a working state such that the repair of component $i$ causes the system to fail. Repair criticality for component $i$ is given in (5).

$$
G_{i}^{R}(\underline{q})=\frac{\partial Q_{S y s}(t)}{\partial p_{i}} .
$$

During a 'noncoherent system analysis' the role of component failure and the role of component repair are considered separately. The overall contribution of component $i$ is then:

$$
G_{i}(\underline{q})=G_{i}^{F}(\underline{q})+G_{i}^{R}(\underline{q}) .
$$

Consider the Boolean expression introduced earlier for the example system; the system unavailability function is given in (2).

As an example calculation for Birnbaum's measure of importance for component $b$, the failure and repair importance are calculated using (4) and (5), and are then summed.

$$
\begin{aligned}
G_{b}^{F}(\underline{q}) & =q_{a} \cdot q_{d}-q_{a} \cdot q_{c} \cdot q_{d}-q_{a} \cdot q_{d} \cdot q_{e}+q_{a} \cdot q_{c} \cdot q_{d} \cdot q_{e} \\
& =q_{a} \cdot q_{d} \cdot\left(1-q_{c}\right) \cdot\left(1-q_{e}\right) ; \\
G_{b}^{R}(\underline{q}) & =q_{a} \cdot q_{c}-q_{a} \cdot q_{c} \cdot q_{d}=q_{a} \cdot q_{c} \cdot\left(1-q_{d}\right) ; \\
G_{b}(\underline{q}) & =q_{a} \cdot q_{d} \cdot\left(1-q_{d}\right) \cdot\left(1-q_{e}\right)+q_{a} \cdot q_{c} \cdot\left(1-q_{d}\right) .
\end{aligned}
$$

\section{The Component Criticality Measure}

For coherent systems, the component criticality measure is defined as: the probability that component $i$ is critical to the system and $i$ has failed, weighted by the system unavailability at time $t, v i z$,

$$
I_{C_{i}}=\frac{G_{i}(\underline{q}) \cdot q_{i}}{Q_{\text {Sys }}(t)} .
$$

When analysis is noncoherent, then component-failure and component-repair can cause SF; hence an expression for failure-importance and repair-importance must be obtained.

For this measure of importance, failure importance $\equiv \operatorname{Pr}\{$ component $i$ is FC to the system; and has failed, weighted by the system unavailability $\}$. Equation (8) is the criticality measure of failure importance:

$$
I_{C_{i}}^{F}=\frac{G_{i}^{F} \cdot q_{i}}{Q_{\text {Sys }}(t)} .
$$

Similarly, repair importance $\equiv \operatorname{Pr}\{$ component $i$ is success (repair) critical to the system; and is in a working state, weighted by the system unavailability $\}$. Equation (9) is the criticality measure of repair importance:

$$
I_{C_{i}}^{R}=\frac{G_{i}^{R} \cdot p_{i}}{Q_{S y s}(t)} .
$$

The overall importance of a component is obtained by summing its failure and repair contributions.

To demonstrate the calculation procedure for this measure, consider the Boolean expression (1). Birnbaum's repair and failure measures have already been calculated and an expression for the system unavailability function has been obtained; 
thus it is a simple matter of substitution to calculate the repair and failure criticality measure for component $b$. From (8) and (9):

$$
\begin{aligned}
I_{C_{b}}^{F} & =\frac{\left[q_{a} \cdot q_{d} \cdot\left(1-q_{c}\right) \cdot\left(1-q_{e}\right)\right] \cdot q_{b}}{Q_{S y s}(t)} ; \\
I_{C_{b}}^{R} & =\frac{\left[q_{a} \cdot q_{c} \cdot\left(1-q_{d}\right)\right] \cdot p_{b}}{Q_{S y s}(t)} ; \\
I_{C_{b}} & =\frac{\left[q_{a} \cdot q_{d} \cdot\left(1-q_{c}\right) \cdot\left(1-q_{e}\right)\right] \cdot q_{b}+\left[q_{a} \cdot q_{c} \cdot\left(1-q_{d}\right)\right] \cdot p_{b}}{Q_{S y s}(t)} .
\end{aligned}
$$

\section{FV MEASURE OF COMPONENT IMPORTANCE}

For coherent systems, the FV measure of component importance [11] is concerned with component failures contributing to SF. This measure is defined as $\operatorname{Pr}\{$ a minimal cs containing component $i$ causes $\mathrm{SF}\}$, and is given in (10).

$$
I_{\mathrm{FV}_{i}}=\frac{\operatorname{Pr}\left\{\bigcup_{k=1 ; i \in C_{K}}^{n_{p}} C_{k}\right\}}{Q_{S y s}(t)} .
$$

This measure can be extended to noncoherent analysis. The FV failure importance $\equiv \operatorname{Pr}\{$ the failed state of component $i$ contributes to SF $\}$; see (11).

$$
I_{\mathrm{FV}_{i}}^{F}=\frac{\operatorname{Pr}\left\{\bigcup_{k=1 ; i \in C_{K}}^{n_{p}} C_{k}\right\}}{Q_{\text {Sys }}(t)} .
$$

Similarly the FV repair importance is defined $\operatorname{Pr}\{$ the working state of component $i$ contributes to SF $\}$, weighted by the system unavailability:

$$
I_{\mathrm{FV}_{i}}^{R}==\frac{\operatorname{Pr}\left\{\bigcup_{k=1 ; \bar{i} \in C_{K}}^{n_{p}} C_{k}\right\}}{Q_{\text {Sys }}(t)} .
$$

The calculation of the FV measure does not involve Birnbaum's measure; instead the prime implicant sets of the system are considered. Consider the Boolean expression in (1).

To calculate the failure importance of component $b$, the prime implicants sets containing $b$ are identified, and then (11) is applied. Similarly, to find the repair importance of component $b$, the prime implicants sets containing $\bar{b}$ are identified, and then (12) is applied.

$$
\begin{aligned}
I_{\mathrm{FV}_{b}}^{F} & =\frac{q_{a} \cdot q_{b} \cdot q_{d}}{Q_{S y s}(t)} ; \\
I_{\mathrm{FV}_{b}}^{R} & =\frac{q_{a} \cdot p_{b} \cdot q_{c}}{Q_{S y s}(t)} ; \\
I_{\mathrm{FV}_{b}} & =\frac{q_{a} \cdot\left(q_{b} \cdot q_{d}+p_{b} \cdot q_{c}\right)}{Q_{\text {Sys }}(t)} .
\end{aligned}
$$

\section{MEASURES OF INITIATOR AND ENABLER IMPORTANCE}

The 2 measures considered in Sections IV and V, the Criticality and the FV measures, are both suitable for system unavailability analysis, and both consider the events which need to exist to fail the system. The Initiator and Enabler measures, on the other hand, analyze the role of component failures in causing and contributing to SF, respectively, and they are both concerned, in a limited capacity, with the sequence of events leading to SF. A component failure can contribute in 1 of 2 ways:

1) it can be an initiating event whereby its occurrence 'when the system is in a critical state' causes system failure,

2) it can be an enabling event whereby its existence permits another, initiator event, to cause SF.

Hence the 2 measures calculate the importance of components in very different roles and are considered separately in Sections VI-A and B.

\section{A. $B \& P$ Initiator Importance}

$\mathrm{B} \& \mathrm{P}$ developed the measure of initiator importance in [12]; the measure is concerned with the failure of components acting as initiating events and thus their occurrence coincides with SF. This measure calculates $\operatorname{Pr}\{$ component $i$ causes SF in $[0, t]\}$. This measure for coherent systems is:

$$
I_{\mathrm{IN}_{i}}=\frac{\int_{0}^{t} G_{i}(\underline{q}) \cdot w_{i}(u) d u}{W_{\text {Sys }}(0, t)} ;
$$

$w_{i}(t) \equiv$ the unconditional failure intensity of component $i$, $W_{S y s}(\mathbb{\#}, t)$ e $s$-expected number of SF in a given interval.

For noncoherent systems, the total initiator importance measure is defined again in 2 terms. Term \#1 is $\operatorname{Pr}\{$ the failure of component $i$ causes SF in the interval $[0, t]\}$ :

$$
I_{\mathrm{IN}_{i}}^{F}=\frac{\int_{0}^{t} G_{i}^{F}(\underline{q}) \cdot w_{i}(u) d u}{W_{\text {Sys }}(0, t)} .
$$

Term \#2 is the initiator repair importance: $\operatorname{Pr}\{$ repair of component $i$ causes system failure in the interval $[0, t]\}$ :

$$
I_{\mathrm{IN}_{i}}^{R}=\frac{\int_{0}^{t} G_{i}^{R}(\underline{q}) \cdot v_{i}(u) d u}{W_{\text {Sys }}(0, t)} .
$$

To calculate the initiator importance of a component, an expression for $W_{\text {Sys }}(0, t)$ must be obtained:

$W_{\text {Sys }}(0, t)$

$$
=\int_{0}^{t}\left[\sum_{i=1}^{n_{F_{i}}} G_{i}^{F}(\underline{q}) \cdot w_{i}(u)+\sum_{i=1}^{n_{R_{i}}} G_{i}^{R}(\underline{q}) \cdot v_{i}(u)\right] d u .
$$

This is not considered in detailed here; for a detailed explanation see [9].

To obtain an expression for $W_{S y s}(0, t)$, it is necessary to calculate Birnbaum's measure for each component (both repair and failure). Having obtained an expression for $W_{\text {Sys }}(0, t)$, it is a simple matter of substitution to calculate the initiator importance of a component; the initiator importance of component $b$ is, using (14) and (15):

$$
\begin{aligned}
I_{\mathrm{IN}_{b}}^{F} & =\frac{\int_{0}^{t}\left[q_{a} \cdot q_{d} \cdot\left(1-q_{c}\right) \cdot\left(1-q_{e}\right)\right] \cdot w_{b} d u}{W_{S y s}(0, t)} ; \\
I_{\mathrm{IN}_{b}}^{R} & =\frac{\int_{0}^{t}\left[q_{a} \cdot q_{c} \cdot\left(1-q_{d}\right) \cdot\right] \cdot v_{b} d u}{W_{\text {Sys }}(0, t)} ; \\
I_{\mathrm{IN}_{b}} & =I_{\mathrm{IN}_{b}}^{F}+I_{\mathrm{IN}_{b}}^{R} .
\end{aligned}
$$




\section{B. Lambert's Enabler Importance}

Lambert introduced the measure of enabler importance in [13], [14]; and is defined, for coherent systems, as $\operatorname{Pr}\{$ the failure of component $i$ allows SF in $[0, t]$ caused by the failure of another component $j$ occurring \}. Lambert developed the expression for this measure:

$$
\begin{aligned}
I_{E_{i}} & =\frac{\sum_{j ; i \neq j ; i, j \in C_{K}} \int_{0}^{t} \Psi(u) \cdot q_{i}(u) \cdot w_{j}(u) d u}{W_{\text {Sys }}(0, t)} \\
\Psi(u) & \equiv Q_{\text {Sys }}\left(1_{i}, 1_{j}, \underline{q}(u)\right)-Q_{\text {Sys }}\left(1_{i}, 0_{j}, \underline{q}(u)\right) ;
\end{aligned}
$$

$i$ is the enabler and $j$ is the initiator.

However, (16) does not exactly specify $\operatorname{Pr}\{$ component $i$ contributes to $\mathrm{SF}$ in a given interval when another component, $j$, causes SF \}. It is an approximation because it does not consider the separate roles of components $i$ and $j$ in causing, or contributing to, SF. For component $i$ to enable SF by the failure of component $j$, then $i$ and $j$ must occur in at least 1 minimal cut-set together, and it must be the existence of 1 such minimal cut-set that causes SF, for $i$ to enable $j$ to initiate failure.

To demonstrate this, consider the following example with minimal CS: $\{$ abe, def, bg, dh .

The Boolean expression for the top event and the system unavailability function are:

$$
\begin{aligned}
T= & a b e+d e f+b g+d h ; \\
Q_{S y s}(t)= & q_{a} \cdot q_{b} \cdot q_{e}+q_{d} \cdot q_{e} \cdot q_{f}+q_{b} \cdot q_{g}+q_{d} \cdot q_{h} \\
& -q_{a} \cdot q_{b} \cdot q_{d} \cdot q_{e} \cdot q_{f}-q_{a} \cdot q_{b} \cdot q_{e} \cdot q_{g} \\
& -q_{a} \cdot q_{b} \cdot q_{d} \cdot q_{e} \cdot q_{h} \\
& -q_{b} \cdot q_{d} \cdot q_{e} \cdot q_{f} \cdot q_{g}-q_{d} \cdot q_{e} \cdot q_{f} \cdot q_{h} \\
& -q_{b} \cdot q_{d} \cdot q_{g} \cdot q_{h} \\
& +q_{a} \cdot q_{b} \cdot q_{d} \cdot q_{e} \cdot q_{f} \cdot q_{g}+q_{a} \cdot q_{b} \cdot q_{d} \cdot q_{e} \cdot q_{g} \cdot q_{h} \\
& +q_{a} \cdot q_{b} \cdot q_{d} \cdot q_{e} \cdot q_{f} \cdot q_{h}+q_{b} \cdot q_{d} \cdot q_{e} \cdot q_{f} \cdot q_{g} \cdot q_{h} \\
& -q_{a} \cdot q_{b} \cdot q_{d} \cdot q_{e} \cdot q_{f} \cdot q_{g} \cdot q_{h} .
\end{aligned}
$$

Let $d$ be an enabler, and $e$ be an initiator; then, for $d$ to act as enabler and contribute to $\mathrm{SF}$ when $e$ actually causes $\mathrm{SF}$, they must be in the same minimal CS. This is minimal CS $2,\{d e f\}$. Therefore, $f$ must fail, and CS 1, 3, 4 must not fail. This means that either $b$ works, or $a$ works from minimal CS 1 ; and $g$ works or $b$ works to prevent minimal CS 3 , and $h$ works to prevent minimal CS 4. Thus the required circumstances are: $f$ and $\overline{a b}$ and $\overline{b g}$ and $\bar{h}$; ie,

$$
f \cdot(\bar{a}+\bar{b}) \cdot(\bar{b}+\bar{g}) \cdot \bar{h}=f \cdot \bar{h} \cdot(\bar{b}+\bar{a} \cdot \bar{g}) .
$$

Expanding this gives

$$
\begin{aligned}
I_{\mathrm{AE}_{d, e}}= & \frac{\int_{0}^{t}\left[q_{f} \cdot\left(1-q_{h}\right) \cdot \Psi_{a, b, g}\right] \cdot q_{d} \cdot w_{e} d u}{W_{S y s}(0, t)}, \\
\Psi_{a, b, g} \equiv & \left(1-q_{b}\right)+\left(1-q_{a}\right) \cdot\left(1-q_{g}\right) \\
& -\left(1-q_{a}\right) \cdot\left(1-q_{b}\right) \cdot\left(1-q_{g}\right) ; \\
I_{\mathrm{AE}_{d, e}}= & \frac{\int_{0}^{t}\left[q_{f} \cdot\left(1-q_{h}\right) \cdot \Psi_{a, b, g}^{*}\right] \cdot q_{d} \cdot w_{e} d u}{W_{S y s}(0, t)} ; \\
\Psi_{a, b, g}^{*} \equiv & 1-q_{a} \cdot q_{b}-q_{b} \cdot q_{g}+q_{a} \cdot q_{b} \cdot q_{g} .
\end{aligned}
$$

$I_{\mathrm{AE}_{i, j}}$ is the actual enabler probability.
The probability calculated using the Lambert formula is:

$$
\begin{aligned}
I_{E_{d, e}}= & \frac{Q_{S y s}\left(1_{d}, 1_{e}, \underline{q}(t)\right)-Q_{\text {Sys }}\left(1_{d}, 0_{e}, \underline{q}(t)\right)}{W_{S y s}(0, t)}, \\
= & \frac{\int_{0}^{t}\left(1-q_{h}\right) \cdot \Psi_{a, b, f, g} \cdot q_{d}(u) \cdot w_{e}(u) d u}{W_{S y s}(0, t)} ; \\
\Psi_{a, b, f, g} \equiv & q_{a} \cdot q_{b} \cdot\left(1-q_{g}\right) \\
& +q_{f} \cdot\left(1-q_{a} \cdot q_{b}-q_{b} \cdot q_{g}+q_{a} \cdot q_{b} \cdot q_{g}\right) .
\end{aligned}
$$

\section{Discussion}

The results in Section VI-B for the Lambert expression and the exact calculation, differ; (16) results in the extra terms:

$$
\left(1-q_{h}\right) \cdot\left[q_{a} \cdot q_{b}-q_{a} \cdot q_{b} \cdot q_{g}\right]=q_{a} \cdot q_{b} \cdot\left(1-q_{g}\right) \cdot\left(1-q_{h}\right) .
$$

These extra terms occur because the measure has failed to consider that components $i$ and $j$ can occur separately in failure combinations, and consequently affect the system state individually as well as jointly; (16) has accounted for the independent role of the initiator but not the enabler.

The required probability is $\operatorname{Pr}\{$ components $i$ and $j$ are critical to system state at time $t$, and component $i$ has failed and component $j$ fails $\}$, all weighted by the $s$-expected number of SF.

To calculate the required probability, first the criticality of component $i$ and $j$ must be calculated; then a correction term, which eliminates the $s$-independent contribution of $i$ and $j$, is required. The criticality of $i$ and $j$ is $\operatorname{Pr}\{$ components $i$ and $j$ are critical to system state $\}, i e$, the system is in a state at time $t$ such that the failure of components $i$ and $j$ would cause SF.

The $\operatorname{Pr}\{$ component $i$ is critical to the system state at time $t$ \} is calculated as:

$$
G_{i}(\underline{q})=Q_{\text {Sys }}\left(1_{i}, \underline{q}(t)\right)-Q_{\text {Sys }}\left(0_{i}, \underline{q}(t)\right),
$$

$Q_{\text {Sys }}\left(1_{i}, \underline{q}(t)\right)$ is $\operatorname{Pr}\{$ system is in a failed state at time $t$, and $i$ is failed $\}$,

$Q_{\text {Sys }}\left(0_{i}, \underline{q}(t)\right)$ is $\operatorname{Pr}\{$ system is in a failed state at time $t$, and $i$ is working $\}$.

Because $Q_{S y s}(t)$ is linear in $q_{i}(t)$, the $G_{i}(\underline{q})$ can be re-written as:

$$
G_{i}(\underline{q})=\frac{\partial Q_{S y s}(t)}{\partial q_{i}(t)}
$$

Hence $\operatorname{Pr}\{$ components $i$ and $j$ are critical to system state $\}$, $G_{i, j}(\underline{q})$ can be obtained by extending this definition of criticality. First, extend the notation:

$Q_{\text {Sys }}\left(1_{i}, 1_{j}, \underline{q}(t)\right)=\operatorname{Pr}\{$ the system is in a failed state with components $i$ and $j$ failed $\}$.

Then $Q_{S y s}\left(1_{i}, 0_{j}, \underline{q}(t)\right)=\operatorname{Pr}\{$ the system is in a failed state with component $i$ failed, and component $j$ working \} .

Then $\operatorname{Pr}\{$ components $i$ and $j$ are critical to the system at time $t\}$ is:

$$
\begin{aligned}
& G_{i, j}(\underline{q})=Q_{\text {Sys }}\left(1_{i}, 1_{j}, \underline{q}(t)\right)-Q_{\text {Sys }}\left(1_{i}, 0_{j}, \underline{q}(t)\right) \\
&-Q_{\text {Sys }}\left(0_{i}, 1_{j}, \underline{q}(t)\right)+Q_{\text {Sys }}\left(0_{i}, 0_{j}, \underline{q}(t)\right) .
\end{aligned}
$$


This is because $Q_{S y s}\left(1_{i}, 1_{j}, q(t)\right)$ represents $\operatorname{Pr}\{$ the system is in a failed state, and components $i$ and $j$ are failed $\}$. To eliminate the individual contributions of components $i$ and $j$, (ie., if they are contained separately in minimal CS, then they make an individual contribution to SF) then $\operatorname{Pr}\{$ the system is failed when $i$ is failed and $j$ is working $\}$, and $\operatorname{Pr}\{$ the system is failed when $i$ is working and $j$ is failed $\}$ is subtracted. Finally, because this subtraction results in an underestimation, the $\operatorname{Pr}\{$ the system is in a failed state and components $i$ and $j$ are working \} must be added.

Because $Q_{S y s}(t)$ is linear in $q_{i}(t)$, the $G_{i, j}$ can be re-written as:

$$
G_{i, j}(\underline{q})=\frac{\partial^{2} Q_{S y s}(t)}{\partial q_{i} \partial q_{j}} .
$$

A correction term to be applied to $G_{i, j}(q)$ can be derived to account for the separate effects of components $i$ and $j$ on the system state. It can be defined as $\operatorname{Pr}\{$ the system is in a critical state for components $i$ and $j$, such that the failure of either $i$ or $j$ alone is sufficient to cause the system to fail $\}$. To calculate the correction term, a modified unavailability function, $Q_{M_{i, j}}(t)$ is required; it considers all minimal CS of the system apart from those containing both $i$ and $j$.

$$
Q_{M_{i, j}}(t)=\bigcup_{k=1 ; i, j \notin C_{K}}^{n_{p}} \operatorname{Pr}\left\{C_{K}\right\} .
$$

The $\operatorname{Pr}\{$ components $i$ and $j$ are critical to the system state such that the failure of either $i$ or $j$ alone would be sufficient to cause the system to fail $\}$ is:

$$
G_{M_{i, j}}(\underline{q})=\frac{\partial^{2} Q_{M_{i, j}}(t)}{\partial q_{i} \partial q_{j}} .
$$

Thus the enabler importance of component $i$, when component $j$ causes SF, is:

$$
I_{E_{i, j}}=\frac{\int_{0}^{t}\left[G_{i, j}(\underline{q})-G_{M_{i, j}}(\underline{q})\right] \cdot q_{i}(t) \cdot w_{j}(u) d u}{W_{S y s}(0, t)} .
$$

The total enabler importance of component $i$ is the sum of the enabler importance of $i$ when component $j$ initiates SF for $j=$ $1, \ldots, n, j \neq i$ is:

$$
I_{E_{i, j}}=\frac{\sum_{j=1, j \neq i}^{n_{F_{j}}} \int_{0}^{t}\left[G_{i, j}(\underline{q})-G_{M_{i, j}}(\underline{q})\right] \cdot q_{i}(t) \cdot w_{j}(u) d u}{W_{S y s}(0, t)} .
$$

Consider the system introduced in (17); the enabler importance of component $d$ when component $e$ causes SF is calculated using this new measure:

$$
\begin{aligned}
\frac{\partial^{2} Q_{S y s}(t)}{\partial q_{d} \partial q_{e}}= & q_{f}-q_{a} \cdot q_{b} \cdot q_{f}-q_{a} \cdot q_{b} \cdot q_{h}-q_{b} \cdot q_{f} \cdot q_{g} \\
& -q_{f} \cdot q_{h}+q_{a} \cdot q_{b} \cdot q_{f} \cdot q_{g} \\
& +q_{a} \cdot q_{b} \cdot q_{g} \cdot q_{h}+q_{a} \cdot q_{b} \cdot q_{f} \cdot q_{h} \\
& +q_{b} \cdot q_{f} \cdot q_{g} \cdot q_{h}-q_{a} \cdot q_{b} \cdot q_{f} \cdot q_{g} \cdot q_{h} .
\end{aligned}
$$

For the modified unavailability function, the minimal CS containing components $d$ and $e$ are extracted to give:

$$
\begin{aligned}
T_{M}= & a b e+b g+d h ; \\
Q_{M_{d, e}}(t)= & q_{a} \cdot q_{b} \cdot q_{e}+q_{b} \cdot q_{g} . \\
& +q_{d} \cdot q_{h}-q_{a} \cdot q_{b} \cdot q_{e} \cdot q_{g} \\
& -q_{a} \cdot q_{b} \cdot q_{d} \cdot q_{e} \cdot q_{h}-q_{b} \cdot q_{d} \cdot q_{g} \cdot q_{h} \\
& +q_{a} \cdot q_{b} \cdot q_{d} \cdot q_{e} \cdot q_{g} \cdot q_{h} ; \\
\frac{\partial^{2} Q_{M_{d, e}}}{\partial q_{d}(t) \partial q_{e}(t)}= & -q_{a} \cdot q_{b} \cdot q_{h}+q_{a} \cdot q_{b} \cdot q_{g} \cdot q_{h} \\
= & q_{a} \cdot q_{b} \cdot q_{h} \cdot\left(q_{g}-1\right) .
\end{aligned}
$$

From (18)

$$
\begin{aligned}
I_{E_{d, e}} & =\frac{\int_{0}^{t}\left[q_{f} \cdot\left(1-q_{h}\right) \cdot \Psi_{a, b, g}\right] \cdot q_{d} \cdot w_{e} d u}{W_{S y s}(0, t)} ; \\
\Psi_{a, b, g} & \equiv 1-q_{a} \cdot q_{b}-q_{b} \cdot q_{g}+q_{a} \cdot q_{b} \cdot q_{g} .
\end{aligned}
$$

This agrees with the required result obtained in Section VI-B.

\section{Extension for Non-Coherent Systems}

This measure can be extended for noncoherent analysis by considering the role that both component failure and working states play in SF. Thus, begin by considering the failure enabler importance of component $i$. The failure of component $i$ could result in component $j$ being either FC or RC.

Thus the probability required for this circumstance: the failure enabler importance of component $i$ when component $j$ causes $\mathrm{SF}$ is:

"The probability that either component $i$ fails, leaving the system FC for component $j$, and component $j$ fails, or that component $i$ fails, leaving the system RC for component $j$, and component $j$ is repaired. Weighted by the $s$-expected number of SF in the interval $[0, t) . "$

The first stage is to calculate the $\operatorname{Pr}\{$ components $i$ and $j$ are FC at time $t\}$ and $\operatorname{Pr}\{$ component $i$ is FC and component $j$ is $\mathrm{RC}$ at time $t\}$. These are in (20) and (21).

$$
\begin{aligned}
& G_{i, j}(\underline{q})=\frac{\partial^{2} Q_{\text {Sys }}(t)}{\partial q_{i}(t) \partial q_{j}(t)} ; \\
& G_{i, \bar{j}}(\underline{q})=\frac{\partial^{2} Q_{\text {Sys }}(t)}{\partial q_{i}(t) \partial p_{j}(t)} .
\end{aligned}
$$

A correction term for each type of criticality is required to ensure that the separate roles of component failures or repairs are eliminated. Thus the first correction term needs to calculate $\operatorname{Pr}\{$ components $i$ and $j$ are $\mathrm{FC}$, such that the failure of either $i$ or $j$ alone is sufficient to cause SF $\}$. Similarly, the second correction term is $\operatorname{Pr}\{$ component $i$ is $\mathrm{FC}$, and component $j$ is $\mathrm{RC}$, such that the failure of component $i$ or the repair of component $j$ alone is sufficient to cause SF $\}$. These are in (22) and (23).

$$
\begin{aligned}
G_{M_{i, j}}(\underline{q}) & =\frac{\partial^{2} Q_{M_{i, j}}(t)}{\partial q_{i}(t) \partial q_{j}(t)} ; \\
G_{M_{i, j}}(\underline{q}) & =\frac{\partial^{2} Q_{M_{i, j}}(t)}{\partial q_{i}(t) \partial p_{j}(t)} .
\end{aligned}
$$


$Q_{M_{i, j}}(t)$ is the modified system unavailability function for $i$ and $j$ is shown next; the union is taken for all prime implicants that do not contain both $i$ and $j$, and also do not contain either $\bar{i}$ or $\bar{j}$.

$$
Q_{M_{i, j}}(t)=\bigcup_{k=1 ; i, j \notin C_{K} ; \bar{i} \notin C_{K} ; \bar{j} \notin C_{K}}^{n_{p}} \operatorname{Pr}\left\{C_{K}\right\} .
$$

$Q_{M_{i, \bar{j}}}(t)$ is the modified system unavailability function for $i$ such that,

$$
Q_{M_{i, j}}(t)=\bigcup_{k=1 ; i, \bar{j} \notin C_{K} ; \bar{i} \notin C_{K} ; j \notin C_{K}}^{n_{p}} \operatorname{Pr}\left\{C_{K}\right\} .
$$

Hence the failure enabler importance of component $i$, when component $j$ initiates SF, is:

$$
\begin{aligned}
I_{E_{i, j}}^{F} & =\frac{\int_{0}^{t}\left[\Psi_{i, j} \cdot q_{i} \cdot w_{j}+\Psi_{i, \bar{j}} \cdot q_{i} \cdot v_{j}\right] d u}{W_{S y s}(0, t)} \\
\Psi_{i, j} & \equiv G_{i, j}(\underline{q})-G_{M_{i, j}}(\underline{q}) \\
\Psi_{i, \bar{j}} & \equiv G_{i, \bar{j}}(\underline{q})-G_{M_{i, \bar{j}}}(\underline{q}) .
\end{aligned}
$$

The total failure enabler importance of component $i$ is:

$$
\begin{aligned}
I_{E_{i}}^{F} & =\frac{\Psi_{1}(u)+\Psi_{2}(u)}{W_{S y s}(0, t)} \\
\Psi_{1}(u) & \equiv \sum_{j=1 ; j \neq i}^{n_{F_{j}}} \int_{0}^{t}\left[G_{i, j}(\underline{q})-G_{M_{i, j}}(\underline{q})\right] \cdot q_{i} \cdot w_{j} d u, \\
\Psi_{2}(u) & \equiv \sum_{j=1 ; j \neq i}^{n_{R_{j}}} \int_{0}^{t}\left[G_{i, \bar{j}}(\underline{q})-G_{M_{i, \bar{j}}}(\underline{q})\right] \cdot q_{i} \cdot v_{j} d u .
\end{aligned}
$$

The probability required for the repair enabler importance of component $i$ when component $j$ causes SF is:

"The probability that either component $i$ has been repaired, leaving the system FC for component $j$, and component $j$ fails, or that component $i$ has been repaired, leaving the system RC for component $j$, and component $j$ is repaired."

By similar arguments to those used to derive the failure enabler importance, the repair enabler importance of component $i$, when component $j$ causes SF, is:

$$
\begin{aligned}
I_{E_{i, j}}^{R}= & \frac{\int_{0}^{t} \Psi(u) d u}{W_{S y s}(0, t)} \\
\Psi(u) \equiv & {\left[G_{\bar{i}, j}(\underline{q})-G_{M_{\bar{i}, j}}(\underline{q})\right] \cdot p_{i} \cdot w_{j} } \\
& +\left[G_{\bar{i}, \bar{j}}(\underline{q})-G_{M_{\bar{i}, \bar{j}}}(\underline{q})\right] \cdot p_{i} \cdot v_{j} .
\end{aligned}
$$

The total repair enabler importance for component $i$ is:

$$
\begin{aligned}
& I_{E_{i}}^{R}=\frac{\Psi_{1}+\Psi_{2}}{W_{S y s}(0, t)} \\
& \Psi_{1} \equiv \sum_{j=1 ; j \neq i}^{n_{F_{j}}} \int_{0}^{t}\left[G_{\bar{i}, j}(\underline{q})-G_{M_{\bar{i}, j}}(\underline{q})\right] \cdot p_{i} \cdot w_{j} d u \\
& \Psi_{2} \equiv \sum_{j=1 ; j \neq i}^{n_{R_{j}}} \int_{0}^{t}\left[G_{\bar{i}, \bar{j}}(\underline{q})-G_{M_{\bar{i}, \bar{j}}}(\underline{q})\right] \cdot p_{i} \cdot v_{j} d u . \quad(27) \\
& G_{\bar{i}, j}(\underline{q}) \quad \quad=\left(\partial^{2} Q_{S y s}(t) / \partial p_{i}(t) \partial q_{j}(t)\right): \operatorname{Pr}\{\text { component } \\
&i \text { is RC, and component } j \text { is FC at time } t\}
\end{aligned}
$$

$$
\begin{aligned}
& G_{\bar{i}, \bar{j}}(\underline{q}) \quad=\left(\partial^{2} Q_{S y s}(t) / \partial p_{i}(t) \partial p_{j}(t)\right): \operatorname{Pr}\{\text { components } \\
& i \text { and } j \text { are RC at time } t\} \text {. } \\
& G_{M_{\bar{i}, j}}(\underline{q})=\left(\partial^{2} Q_{M_{\bar{i}, j}}(t) / \partial p_{i}(t) \partial q_{j}(t)\right): \operatorname{Pr}\{\text { component }
\end{aligned}
$$

Again consider the Boolean expression in (1) whose system unavailability function is in (2). The enabler importance of component $b$ is calculated when component $c$ causes SF. The first step is to obtain the expression for the 4 modified unavailability functions required.

$$
\begin{aligned}
Q_{M_{b, c}=} & q_{a} \cdot q_{b} \cdot q_{d}+q_{a} \cdot q_{c} \cdot q_{d}+q_{a} \cdot q_{d} \cdot q_{e} \\
& -q_{a} \cdot q_{b} \cdot q_{c} \cdot q_{d}-q_{a} \cdot q_{b} \cdot q_{d} \cdot q_{e}-q_{a} \cdot q_{c} \cdot q_{d} \cdot q_{e} \\
& +q_{a} \cdot q_{b} \cdot q_{c} \cdot q_{d} \cdot q_{e} ; \\
Q_{M_{b, \bar{c}}=} & q_{a} \cdot q_{b} \cdot q_{d} \cdot+p_{c} \cdot q_{d} \cdot q_{e}+q_{a} \cdot q_{d} \cdot q_{e} \\
& -q_{a} \cdot p_{c} \cdot q_{d} \cdot q_{e}-q_{a} \cdot q_{b} \cdot q_{d} \cdot q_{e} ; \\
Q_{M_{\bar{b}, \mathrm{c}}}= & q_{a} \cdot q_{c} \cdot q_{d}+q_{a} \cdot q_{d} \cdot q_{e}-q_{a} \cdot q_{c} \cdot q_{d} \cdot q_{e} ; \\
Q_{M_{\bar{b}, \bar{c}}}= & p_{c} \cdot q_{d} \cdot q_{e}+q_{a} \cdot q_{d} \cdot q_{e}-q_{a} \cdot p_{c} \cdot q_{d} \cdot q_{e} .
\end{aligned}
$$

Now

$$
\begin{aligned}
G_{b, c}(\underline{q}) & =-q_{a} \cdot q_{d}+q_{a} \cdot q_{d} \cdot q_{e}, \\
G_{M_{b, c}}(\underline{q}) & =-q_{a} \cdot q_{d}+q_{a} \cdot q_{d} \cdot q_{e} ; \\
G_{b, \bar{c}}(\underline{q}) & =0, \quad G_{M_{b, \bar{c}}}(\underline{q})=0 ; \\
G_{\bar{b}, c}(\underline{q}) & =q_{a}-q_{a} \cdot q_{d}, \quad G_{M_{\bar{b}, c}}(\underline{q})=0 ; \\
G_{\bar{b}, \bar{c}}(\underline{q}) & =0, \quad G_{M_{\bar{b}, \bar{c}}}(\underline{q})=0 .
\end{aligned}
$$

Then from (24) and (25):

$$
\begin{aligned}
I_{E_{b, c}}^{F} & =0 \\
I_{E_{b, c}}^{R} & =\frac{\int_{0}^{t} q_{a} \cdot\left(1-q_{d}\right) \cdot p_{b}(u) \cdot w_{c}(u) d u}{W_{S y s}(0, t)} .
\end{aligned}
$$

Note: Components $b$ and $c$ or $b$ and $\bar{c}$ do not appear in any of the prime implicants together; hence the failure enabler importance of $b$ when $c$ causes system-failure is zero. Also, only components $\bar{b}$ and $c$ occur together in the prime implicant sets, thus as anticipated, the term involving them is nonzero.

\section{APPLICATION TO A LEAK-PROTECTION SYSTEM}

To demonstrate the results that the application of these noncoherent measures provide, consider the leak protection system in Fig. 1.

High-pressure gas flows through the pipes as part of a transmission system. A gas leak can occur beyond the isolation valve, due to a leak in either the pipe itself or at a flange. A gas-detection system detects a gas leak, and to simplify the analysis, it is assumed to be perfectly reliable. Upon gas detection, the isolation unit (which consists of the isolation valve and its controller) closes the isolation valve, thus preventing the gas from flowing through the valve and out of the damaged pipe. However, if isolation is successful, the high-pressure gas will cause a hammer effect on the valve, which can rupture the pipe in front of the 


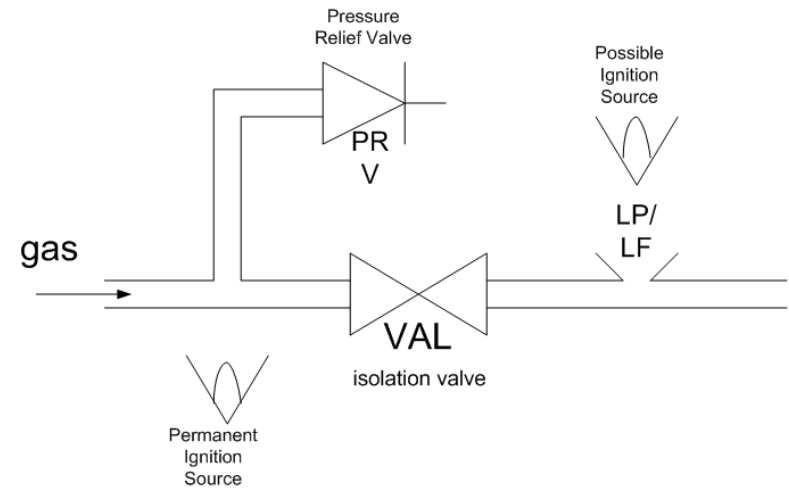

Fig. 1. Leak protection system.

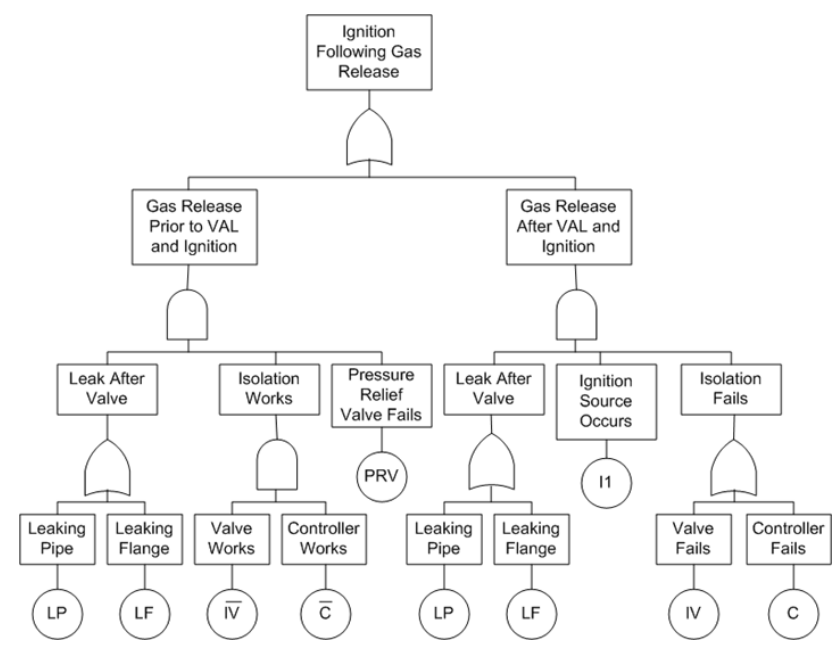

Fig. 2. Fault-tree representing the possible causes of the top-event for the system in Fig. 1.

valve. Due to the permanent ignition source located in this section, it is essential to divert the gas flow elsewhere and to prevent the pipe from rupturing; thus a pressure relief valve has been installed.

The system is designed to protect against an ignition following a gas release; hence the system is in a failed state if a gas leak occurs and an ignition results. Fig. 2 shows a FT, which represents the possible causes of the top event: an ignition source following a gas leak.

From this FT in Fig. 2, the prime implicant sets of the system can be obtained using a bottom-up approach and applying the consensus theorem to the implicant sets obtained. The 8 primeimplicant sets of the system are:

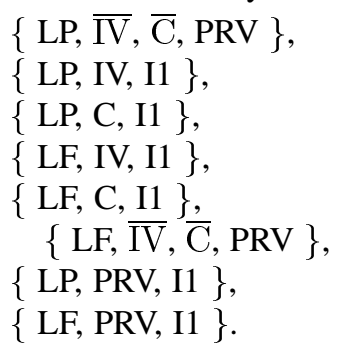

Prior to calculating the various measures of importance for all the components, the role that each component failure/repair could have in a system-failure must be considered. Then the appropriate measures of importance can be used to analyze each
TABLE I

REPAIR AND FAILURE RATES, AND INSPECTION INTERVALS, FOR THE SYSTEM COMPONENTS

\begin{tabular}{r|ccc} 
Component & $\begin{array}{c}\text { Failure Rate } \\
\text { (per hour) }\end{array}$ & $\begin{array}{c}\text { MTTR } \\
\text { (hour) }\end{array}$ & $\begin{array}{c}\text { Inspection } \\
\text { Interval } \\
\text { (hour) }\end{array}$ \\
\hline LF & $1.8 \cdot 10^{-6}$ & 1 & 0 \\
LP & $2 \cdot 10^{-8}$ & 1 & 0 \\
IV & $17.3 \cdot 10^{-6}$ & 20 & 8760 \\
C & $5 \cdot 10^{-6}$ & 12 & 8760 \\
I1 & $\begin{array}{c}\text { Occurs } 1 / \text { week and } \\
\text { lasts for } 12 \text { minutes } \\
17.3 \cdot 10^{-6}\end{array}$ & N/A & N/A \\
PRV & 170 & 8760 \\
\hline
\end{tabular}

TABLE II

COMPONENT UNAVAILABILITY AND UNCONDITIONAL FAILURE AND REPAIR RATES

\begin{tabular}{r|ccc} 
Component & Unavailability & $\begin{array}{c}\text { Unconditional } \\
\text { failure rate }\end{array}$ & $\begin{array}{c}\text { Unconditional } \\
\text { repair rate }\end{array}$ \\
\hline LF & $1.8 \cdot 10^{-6}$ & $1.799 \cdot 10^{-6}$ & $1.8 \cdot 10^{-6}$ \\
LP & $2 \cdot 10^{-8}$ & $1.998 \cdot 10^{-8}$ & $2 \cdot 10^{-8}$ \\
IV & 0.07612 & $1.598 \cdot 10^{-5}$ & 0.003806 \\
C & 0.2196 & $3.902 \cdot 10^{-6}$ & 0.0183 \\
I1 & $1 / 840$ & $1 / 840$ & $\mathrm{~N} / \mathrm{A}$ \\
PRV & 0.07612 & $1.598 \cdot 10^{-5}$ & 0.003806 \\
\hline
\end{tabular}

component's contribution to SF. Both the isolation unit and the pressure relief valve are safety systems; so they are passive components and hence their failed and working (in the case of the isolation unit) states can only act as enablers. The failure of the remaining 3 components can act as either initiators or enablers. Consider the situation whereby a gas leak exists at time $t$, and the isolation unit has failed prior to time $t$, the system will be in a failed state if an ignition source occurs before the leak is repaired. In this case the gas leak is an enabling event and the ignition source is the initiating event. Similarly if an ignition source exists at some time $t$ and the isolation unit has failed prior to time $t$, then the occurrence of a gas leak while the ignition source is present, will cause the system top event. Thus the gas leak is the initiating event and the ignition source is an enabling event.

This shows that, of the measures introduced, only the FV measure, the measure of criticality, and the measure of enabler importance, should be used to analyze the importance of safety system components. On the other hand, all 5 measures of importance can be used to analyze the importance of the remaining 3 components.

Appropriate failure and repair rates have been assigned to each component as given in Table I. Inspection intervals have been assigned to the valves, because it is standard to inspect such dormant components in order to reveal a failure; in this case they are inspected annually. From these rates, the component unavailability and the unconditional failure and repair rates are calculated from (26). The results are in Table II.

$$
\begin{aligned}
q_{\mathrm{AV}} & =\lambda \cdot\left(\tau+\frac{\theta}{2}\right) ; \\
w & =\lambda \cdot(1-q) ; \\
v & =\frac{q}{\tau}
\end{aligned}
$$


TABLE III

RESULTS FOR THE VARIOUS IMPORTANCE MEASURES

\begin{tabular}{r|rrrrr} 
Event & Birnbaum's & Criticality & FV & Initiator & Enabler \\
\hline LF & $0.0551(2)$ & $0.9875(4)$ & $0.98997(4)$ & $0.9571(1)$ & $5.79 \cdot 10^{-3}(5)$ \\
LP & $0.0552(1)$ & $0.01097(5)$ & $0.01099(5)$ & $0.01063(2)$ & $6.66 \cdot 10^{-5}(8)$ \\
I1 & $5.08 \cdot 10^{-7}(4)$ & $6.05 \cdot 10^{-3}(6)$ & $7.17 \cdot 10^{-3}(6)$ & $5.83 \cdot 10^{-3}(3)$ & $5.7 \cdot 10^{-3}(6)$ \\
IV & $1.56 \cdot 10^{-9}(8)$ & $1.18 \cdot 10^{-3}(8)$ & $1.64 \cdot 10^{-3}(8)$ & N/A & $0.9976(3)$ \\
IV & $1.08 \cdot 10^{-7}(6)$ & $0.9927(3)$ & $0.99398(2)$ & N/A & $0.9621(4)$ \\
C & $1.84 \cdot 10^{-9}(7)$ & $6.01 \cdot 10^{-3}(7)$ & $4.74 \cdot 10^{-3}(7)$ & N/A & $0.9998(2)$ \\
C & $1.26 \cdot 10^{-7}(5)$ & $0.9929(2)$ & $0.99398(2)$ & N/A & $4.35 \cdot 10^{-4}(7)$ \\
PRV & $1.31 \cdot 10^{-6}(3)$ & $0.99398(1)$ & $0.9944(1)$ & N/A & $0.9999(1)$ \\
\hline
\end{tabular}

(The equation number is provided for each result) IV $\equiv$ failure of IV; $\overline{\mathrm{IV}} \equiv$ repair of IV; $\mathrm{C} \equiv$ failure of $\mathrm{C}$; $\overline{\mathrm{C}} \equiv$ repair of $\mathrm{C}$; $\mathrm{PRV} \equiv$ failure of PRV

\section{RESULTS}

Birnbaum's measure of repair and failure importance was calculated for all components to enable calculation of the $s$-expected number of SF in a given interval. Table III shows the results. The system is most likely to be in a critical state for LP and least likely to be in a critical state for IV. The numerical difference for Birnbaum's importance of the LP and LF is very small; thus the system is most likely to be in a critical state for a leak in the pipe-work, (either pipe or flange). Thus: if system performance is inadequate, then extra resources should be allocated to:

- Reduce existence of the necessary and sufficient conditions for the leaking pipe or leaking flange to be critical.

The conclusions here are limited because there is no real distinction between the 2 possible leaks: LP and LF. The criticality measure can be used to analyze which of the 2 components is most likely to be in a failed state when the system is failed. The results highlight that the system is most likely to be in a failed state due to LF. Thus the previous conclusion about resource allocation can be made more specific. The main priority should lie with:

- Reduction of the existence of the necessary and sufficient conditions for the system to be in a critical state for the LF.

- Increase the reliability of the flange.

The criticality measure also highlights that of the two safety systems: it is the pressure relief valve that is most likely to be in a failed state when the system is failed, thus if resources are to be allocated to safety system improvement, efforts should be concentrated on the PRV.

The results obtained from the initiator importance, in Table III, again confirm these conclusions. LF is most likely to cause SF. LP is ranked second and I1 is the least likely event to cause SF.

The FV measure ranks the contribution each component failure/repair makes to SF. The results are in Table III. The top 3 ranked events are: PRV, $\overline{\mathrm{IV}}$, and $\overline{\mathrm{C}}$. Thus the isolation unit is the most reliable of the 2 safety systems; this conclusion agrees with that from the criticality results. Thus if safety systems are to be improved, then the PRV should be done first. The isolation valve is slightly more reliable than the controller. Hence efforts to improve the isolation unit should be concentrated on the controller. The FV measure also highlights that a leak in the flange is more likely to contribute to SF than a leak in the pipe. This again confirms that the flange is less reliable than the pipe itself.
Finally, the results obtained from the enabler measure are considered. The failure of PRV is ranked highest; thus it is most likely to be in a failed state at time $t$, and enable a component which can act as an initiator to fail and cause SF. The components of $\mathrm{C}$ and IV, and enabling system failure are ranked 2nd and 3rd, again highlighting that it is the more reliable of the 2 safety systems. Also as anticipated, due to component reliabilities and the prime implicants sets of the system, the controller is more likely to fail and enable SF than the isolation valve.

It is essential to consider importance measures in their entirety; upgrading worst case, and reconsidering system importance, is not to be used because it can lead to alternating system design.

\section{REFERENCES}

[1] J. D. Andrews and T. R. Moss, Reliability and Risk Assessment, 2nd ed: Professional Engineering Publishing, 2002.

[2] Kumamoto and E. J. Henley, Reliability Engineering and Risk Assessment: Prentice Hall, 1981.

[3] A. Bendall and J. Ansell, "The incoherency of multistate coherent systems," Reliab. Eng., vol. 8, pp. 165-178, 1984.

[4] R. E. Barlow and F. Proschan, Mathematical Theory of Reliability: Wiley, 1965.

[5] J. D. Andrews, "To not or not to not," in Proc. Int. System Safety Conf., Sept., pp. 267-275.

[6] Inagaki and E. J. Henley, "Probabilistic evaluation of prime implicants and top-events for noncoherent systems," IEEE Trans. Rel., vol. R-29, no. 5, Dec. 1980.

[7] Becker and Camarinopoulos, "Failure frequencies of noncoherent structures," Reliab. Eng. Syst. Saf., vol. 41, pp. 209-215, 1993.

[8] P. S. Jackson, "On the s-importance of elements and implicants of noncoherent systems," IEEE Trans. Rel., vol. R-32, no. 1, Apr. 1983.

[9] S. Beeson and J. D. Andrews, "Birnbaum's measure of component importance for noncoherent systems," IEEE Trans. Rel., vol. 52, no. 2, June 2003

[10] Z. W. Birnbaum, "On the importance of different components in a multicomponent system," in Multivariate Analysis, P. Krishnaiah, Ed: Academic Press, 1969.

[11] J. Fussell, "How to hand calculate system reliability characteristics," IEEE Trans. Rel., vol. R-24, pp. 169-174, Aug. 1975.

[12] Barlow and Proschan, "Importance of system components and fault tree events," Stochastic Processes and Their Applications, vol. 3, pp. $153-173,1975$.

[13] H. E. Lambert, "Fault Trees for Decision Making in Systems Analysis," Ph.D., Univ. of California, Livermore.

[14] _ , "Measures of importance of event and cut sets in fault trees," in Reliability and Fault Tree Analysis: Theoretical and Applied Aspects of System Reliability and Safety Assessment: SIAM, pp. 77-100.

[15] J. D. Andrews and S. J. Dunnett, "Improving accuracy in event tree analysis," in Foresight and Precaution, Cottam, Harvey, Pape, and Tate, Eds.

[16] - "Event tree analysis using binary decision diagrams," IEEE Trans. Rel., vol. 49, no. 2, June 2000. 
Sally Beeson graduated from Loughborough University in 1999 with a firstclass honors degree. She has continued her studies at Loughborough by undertaking research into risk and reliability quantification methods-in particular, on the analysis of noncoherent systems using binary decision diagrams. She has been involved in developing new importance measures for noncoherent systems, and how these can be used in efficient calculation of system-failure frequency.
John D. Andrews is a Professor in the Department of Systems Engineering at Loughborough University. He recently transferred to this department following his initial appointment to the Department of Mathematical Sciences in 1989. Prior to this, John had 9 years of industrial-research with British Gas, and 2 years lecturing in the Mechanical Engineering Department at the University of Central England. His current research interests are in the assessment of the safety and risk of potentially hazardous industrial activities. This research has been heavily supported by industrial funding. In recent years, grants have been secured from the MOD, Rolls Royce Aero Engines, Mobil North Sea, and Bechtel. He has numerous journal/conference publications along with a jointly authored book Reliability and Risk Assessment (now in its second edition). 